\title{
Online social networking and psychological experiences: The perceptions of young people with mental health difficulties.
}

\author{
Amy Singletona, Paul Abelesb and Ian C. Smitha
}

\author{
A. Doctorate in Clinical Psychology, C27 Furness College, \\ Lancaster University, Lancaster, LA1 4YG ${ }^{1}$ \\ B. Central Manchester University Hospitals NHS Foundation Trust \\ Harrington Building, Royal Manchester Children's Hospital, \\ Oxford Road, M13 9WL
}




\begin{abstract}
Objectives: This study explores the interaction between online social networking experiences and wellbeing in 12 young people accessing mental health services.

Methods: Data from semi-structured interviews was analysed using Grounded Theory methodology.

Results: “Threats and judgement” and “connection and support” were experienced by adolescents, facilitated by having continuous access to a vast social network. These experiences influenced adolescents’ psychological wellbeing, mediated by their responses to threat and judgement and maintaining "safe sharing” with their network. Social network use was conceived as a gamble of balancing its potentially positive and negative impact in a culture in which social network use appears to be unavoidable.
\end{abstract}

Conclusions: The findings indicate the importance of routine assessment and formulation of social networking use in understanding adolescents’ psychological distress. Furthermore, a range of opportunities exist for clinicians to utilise the anonymity and peer support that social networks offer to broaden the range of mental health services offered to young people.

Keywords: social media, online social networks, mental health, adolescents, qualitative, grounded theory 


\section{Introduction}

Adolescence is considered a critical period for young people's physical, cognitive and social development (Steinberg, 2010). With the rise of the internet and social networking sites (SNS), in the developed world there has been an unprecedented shift in adolescents' patterns of social interaction and the culture in which they live. Twelve to fifteen year olds in the UK now spend an average of 18.9 hours online each week, a figure which has more than doubled since 2005 (Ofcom, 2015). A review in 2013 found that, in the UK specifically, 85\% of 12-15 year olds access their main SNS profile every day and 20\% do so more than ten times a day, facilitated by the increasing use of mobiles and tablets to gain access (Ofcom, 2013).

Adolescence also marks a time in which there is a sharp increase in the prevalence of mental health difficulties. For example, an epidemiological study in the US (Merikangas et al., 2010) indicated that over 45\% of 13-18 year olds have experienced a mental health difficulty in their lifetime, with over $20 \%$ of these being classed as a "severe disorder".

\subsection{The challenges and opportunities associated with SNS use}

Research has responded to the growth and spread of social networking in recent years, exploring how SNS are used by different groups (e.g. Gosling, Augustine, Vazire, Holtzman, \& Gaddis, 2011; Lawlor \& Kirakowski, 2014) and the benefits and risks associated with SNS (e.g. Staksrud, Ólafsson, \& Livingstone., 2013; Ahn \& Shin, 2013; Müller et al., 2016). In relation to adolescents’ wellbeing, SNS have been found to offer both challenges and opportunities.

\subsubsection{Social relationships}

In their nature as tools for socialising, SNS offer access to the personal information and daily activities of a vast number of others in a way that has never previously been 
possible. The process of seeking and sharing information has been the interest of many authors, often in relation to the "online disinhibition effect", the finding that individuals typically disclose more personal information and seek more private information from others than they would offline (Suler, 2004).

Social relationships can act as a protective factor against mental health difficulties through providing emotional support (Chu, Saucier, \& Hafner, 2010) and creating a sense of belonging and wellbeing (Quinn \& Oldmeadow, 2013). For example, a longitudinal study by Rafnsson et al. (2015) found that the size of, and frequency of contact with, their social network predicted an individual's future wellbeing. In contrast, abusive or rejecting relationships can influence young people's beliefs about themselves as lovable or worthwhile people and can violate the norms of trust, respect and reciprocity expected in social relationships (Bretherton, Munholland, Cassidy, \& Shaver, 1999).

There is ongoing debate about whether SNS strengthen or erode social relationships. SNS use has been found to interfere with typical family activities, including impairing parentchild communication (Subrahmanyam \& Greenfield, 2008). Connecting with others on SNS has been found to have no impact on overall social isolation (Ahn \& Shin, 2013), can lead to the avoidance of problems in the "real world" (Kim, LaRose, \& Peng, 2009) and to a lack of quality connections offline, which in turn has been linked to depression and further social isolation (O’Keeffe \& Clarke-Pearson, 2011). Nevertheless, SNS can also facilitate involvement in community activities (O’Keeffe \& Clarke-Pearson, 2011), and, for some, the opportunity to experience social support in a way that they might have previously been unable to access (Ofcom, 2013).

\subsubsection{Identity development}

Through social relationships, adolescents learn to adjust their self-presentation based upon the reactions of others, which begins a process of rehearsing and integrating those 
elements of the self that will ultimately form their social identity (Schlenker, 1986). Indeed, one study found that in their offline social networks, adolescents formed relationships with those who had similar degrees of hopefulness (Parker et al., 2015). If, as developmental theorists suggest, young people are more likely to integrate aspects of themselves into their identity that are positively responded to by others (Steinberg, 2008) the continuous feedback from other users on SNS might be central to their developing identities.

\subsubsection{Online communication and associated risks}

Valkenburg and Peter (2011) suggest that online communication facilitates and encourages the sharing of personal information through three unique features, "anonymity" (no immediate feedback to communication), “asynchronization” (editing and conscious consideration of the information offered), and "accessibility" (the opportunity to interact with a vastly increased number of people ). The anonymity of the internet has been associated with an array of risks for young people, including “grooming”, exposure to sexual content, “surveillance behaviour” in romantic relationships and cyberbullying (Muise, Christofides, \& Desmarais, 2009; Staksrud et al., 2013). Researchers suggest that the nature of online communication serves to increase the risk of cyberbullying through encouraging disinhibition and deindividuation as there is no capacity for the perpetrator to witness the victim's response (Cassidy, Faucher, \& Jackson, 2013). A recent review of studies suggests that around one quarter of adolescents have experienced cyber-bullying (Hamm et al., 2015), experiences that have been associated with depression, anxiety, severe isolation, and suicidal ideation (Hamm et al., 2015; Hinduja \& Patchin, 2010).

\subsubsection{Mental health}

SNS use has been associated with correlates of psychological distress in young people; for example, adolescents' intense use of SNS correlates with low self-esteem (Müller et al., 2016). The availability of information about others' lives and activities also encourages 
negative social comparisons in which young people believe that others' lives are happier and better than their own (Chou \& Edge., 2012) contributing to depressive symptoms through rumination (Feinstein et al., 2013). SNS are increasingly used for posts relating to selfharming behaviours and suicidal ideation (Lewis, Heath, Michal, \& Duggan, 2012), although further research is needed to clarify the relationship between expressed ideation and at-risk behaviours (Cash, Thelwall, Peck, Ferrell, \& Bridge, 2013).

\subsubsection{Opportunities}

Despite the possible risks of SNS, SNS use has also been associated with positive outcomes including increased creativity, self-expression (Collin, Rahilly, Richardson, \& Third, 2011) and peer relationships with previously inaccessible groups, which in turn may enhance wellbeing (Spies Shapiro \& Margolin, 2013; Ahn \& Shin, 2013). As the risks and opportunities associated with SNS are thought to be interdependent (Collin et al., 2011), it is necessary to help young people develop skills to manage the risks so they may benefit from the positive opportunities that SNS can offer.

\subsection{Clinical populations}

There is currently a dearth of research in areas which might help us better understand the complex relationship between SNS use and psychological distress. The SNS experiences of clinical populations are under-researched (Anderson, Fagan, Woodnutt, \& ChamorroPremuzic, 2012), and these may differ from those of the general population. In addition, research into SNS use has tended to focus on the links between use and pre-defined psychosocial concepts. Given the complexity of this phenomenon, qualitative research which explores people's lived experiences of SNS and perceptions of its impact upon their psychological health will help provide us with a more complete picture of the processes at work. In clinical populations, such knowledge will also help clinicians identify the 
"protective” and "risk” facets of SNS use and assist in planning and delivering effective psychological interventions.

\subsection{Aims}

In this study we explore how young people with mental health difficulties perceive the relationship between their SNS use and their psychological and emotional wellbeing. Our aim was to develop an initial theoretical model based on the young people's understanding of how their SNS interactions might impact upon their distress and wellbeing. A grounded-theory informed analysis was utilised to generate this model. Our primary research question was "What are young people’s perceptions of how SNS use interacts with their wellbeing and distress?” Within this broad question we were also interested in addressing the secondary question "How do young people use SNS for self-disclosure and self-presentation in relation to their emotional experiences?”

\section{Method}

\subsection{Participants}

\subsubsection{Recruitment strategy}

Participants were recruited from community child and adolescent mental health services in England. Inclusion criteria were:

- $\quad$ Aged 13-18 years

- Accessing mental health services

- English as first language

- Consent provided by themselves or parent/guardian (if under 16)

\subsubsection{Recruited participants}


Twelve participants were recruited who met the inclusion criteria and gave consent. Table 1 illustrates the demographic features of the participants. 
Table 1.

Characteristics of Participants and Their SNS Usage

\begin{tabular}{|c|c|c|c|c|c|}
\hline Pseudonym & Age & Gender & SNS Used & $\begin{array}{l}\text { No. of years } \\
\text { using SNS }\end{array}$ & Frequency of use of SNS \\
\hline 1. Sally & 16 & Female & $\begin{array}{c}\text { Facebook, Twitter, Instagram, } \\
\text { Tumblr, Pinterest }\end{array}$ & 5 & More than once an hour \\
\hline 2. Lizzie & 16 & Female & Facebook, Twitter, Ask.fm & 7 & Daily or every other day \\
\hline 3. Jane & 15 & Female & Facebook, Tumblr & 4.5 & Daily or every other day \\
\hline 4. Simon & 14 & Male & $\begin{array}{l}\text { Facebook, Twitter, Blackberry } \\
\text { Messenger, Whatsapp, Instagram, } \\
\text { Moodswing, Snapchat, Vine, } \\
\text { Meetme }\end{array}$ & 3 & $\begin{array}{l}\text { Some SNS daily, some } \\
\text { monthly, some continuous }\end{array}$ \\
\hline 5. Jules & 13 & Female & $\begin{array}{l}\text { Facebook, Snapchat, Instagram, } \\
\text { Tumblr }\end{array}$ & 4 & Continuous \\
\hline 6. Ruby & 17 & Female & $\begin{array}{l}\text { Facebook, Tumblr, Twitter, } \\
\text { Whatsapp, Kik }\end{array}$ & 6 & More than once an hour \\
\hline 7. Nina & 13 & Female & $\begin{array}{l}\text { Facebook, Blackberry Messenger, } \\
\text { MySpace, Twitter, Instagram, } \\
\text { Snapchat }\end{array}$ & 7 & $\begin{array}{l}\text { Continous when not at } \\
\text { school }\end{array}$ \\
\hline 8. Kim & 17 & Female & $\begin{array}{c}\text { Facebook, Twitter, Blackberry } \\
\text { Messenger }\end{array}$ & 6 & Every hour \\
\hline 9. Sarah & 18 & Female & $\begin{array}{l}\text { Facebook, Pinterest, Twitter, } \\
\text { Myspace, Bebo }\end{array}$ & 7 & Continuous \\
\hline 10. Montague & 14 & Male & Facebook, Youtube & 3 & Every day \\
\hline
\end{tabular}




\begin{tabular}{cccccc}
\hline 11. Natalie & 15 & Female & $\begin{array}{c}\text { Facebook, Instagram, Blackberry } \\
\text { Messenger, Snapchat }\end{array}$ & 5 & Continuous \\
\hline 12. Ben & 16 & Male & Facebook & 1 & $\begin{array}{c}\text { Continuous when not at } \\
\text { school }\end{array}$ \\
\hline
\end{tabular}




\subsection{Design and materials}

A qualitative design using semi-structured interviews was adopted and, as we were interested in participant perceptions, a constructivist grounded theory-informed approach to analysis was used. Interviews in this context are intended to “elicit views of the person's subjective world” (Charmaz, 2006, p.29) and use a flexible approach. Whilst we developed and made use of a 'topic guide' to provide structure to the interviews, this was regularly adapted in response to emerging issues and ideas across interviews to inform and test the emerging theory.

Consultation was sought from a service-user group of young people who had accessed mental health services, who advised on the content and format of the information pack documents and the initial topic guide. The initial interview topic guide included broad questions including “Tell me about your experiences of using social networking sites” and “Does using online social networks impact how you feel?” In later interviews, these questions were adapted to assess and explore dimensions that had been highlighted in earlier interviews, for example "Do you ever talk about your feelings or difficulties when you use social networking sites?” and “Do you ever compare yourself to others online?”

\subsection{Procedure}

After peer review and receipt of the necessary ethical approval, participants were recruited either via clinicians or through posters and "business cards" displayed in the services’ waiting rooms. Potential participants could opt-in using a reply slip, by telephone, by email or could ask another person contact the researchers for them. After opt-in, an initial contact was made to allow participants the opportunity to ask questions about the study and to collect demographic information which facilitated theoretical sampling. Each young person was supplied with a recruitment pack with information about the study. Permission 
was sought from the young person’s parent/guardian prior to interview. Participants were made aware that they were able to opt-out of the study at any time.

Participants were interviewed for up to two hours at a place of their choosing. A debrief sheet was given to participants following the interview. Interviews were digitally recorded and transcribed, with data stored securely at the university sponsoring the research.

\subsection{Data analysis}

In grounded theory methodology, analysis occurs alongside data collection (Charmaz, 2006). After each third interview, the data was transcribed and analysed. This analysis consisted of initial "line-by-line" coding. These were then grouped into "focused" codes, which represented the most frequently occurring initial codes. Throughout the transcription and coding process, “memos” were recorded, described by Charmaz as the process in which "you stop and analyze your ideas about the codes in any-and-every-way that occurs to you during the moment” (Charmaz, 2006, p. 72). After the analysis of each "group" of interviews, the focused codes were triangulated with memos to produce conceptual codes, which provided a brief narrative of frequent themes in the interviews and "gaps" in understanding the data. Considering conceptual codes in relation to each led to the development of an initial theoretical model. A process of constant comparison was employed where new data were frequently compared to the existing findings and emerging framework in order to enrich or adjust the understanding being developed. Theoretical sampling in subsequent interviews and amendments to the topic guide were used in order to test hypotheses, clarify, expand or enrich the understanding being generated.

\subsubsection{Validity and integrity of analysis}

Audio-recorded interviews were listened to independently by each author to identify any assumptions evident in the interviewer's line of questioning. Two of the authors 
independently produced codes for a sample of the data and compared these, negotiating any discrepancies and modify the codes and interview topic guide to enhance inter-rater reliability. The theoretical model was developed by all authors and each contributed to theoretical sampling decisions.

\subsection{Reflexivity}

The authors are three practising clinical psychologists who have an interest in mental health and psychological distress both clinically and within research. The first author developed an interest in this area from therapeutic work in which young people’s SNS experiences were central to the formulation and intervention processes within therapy. The second and third authors have contrasting experiences. One did not have extensive experience of working in children's services or other recent contact with adolescents in other roles but had experience utilising grounded theory methodology. The other had extensive experience working within children's mental health services and in conducting research using computerised technologies. Reflective diaries and supervision were used to minimise subjective experiences and beliefs on interpretation of the data, such as previous biases regarding the "superior" value of offline communication.

\section{Findings}

Analysis of the data identified two key mechanisms influencing adolescents’ wellbeing: “threats and judgement” and “connection and support”, resulting from having "continuous access to a vast social network”. Several factors contributed to how these mechanisms influenced wellbeing, including responses to threat and the processes and outcomes of "safe sharing”. Ultimately adolescents' interactions with SNS were conceived as a gamble of balancing the positives and negatives of their experiences in a culture in which SNS use felt unavoidable. Figure 1 illustrates these findings. 


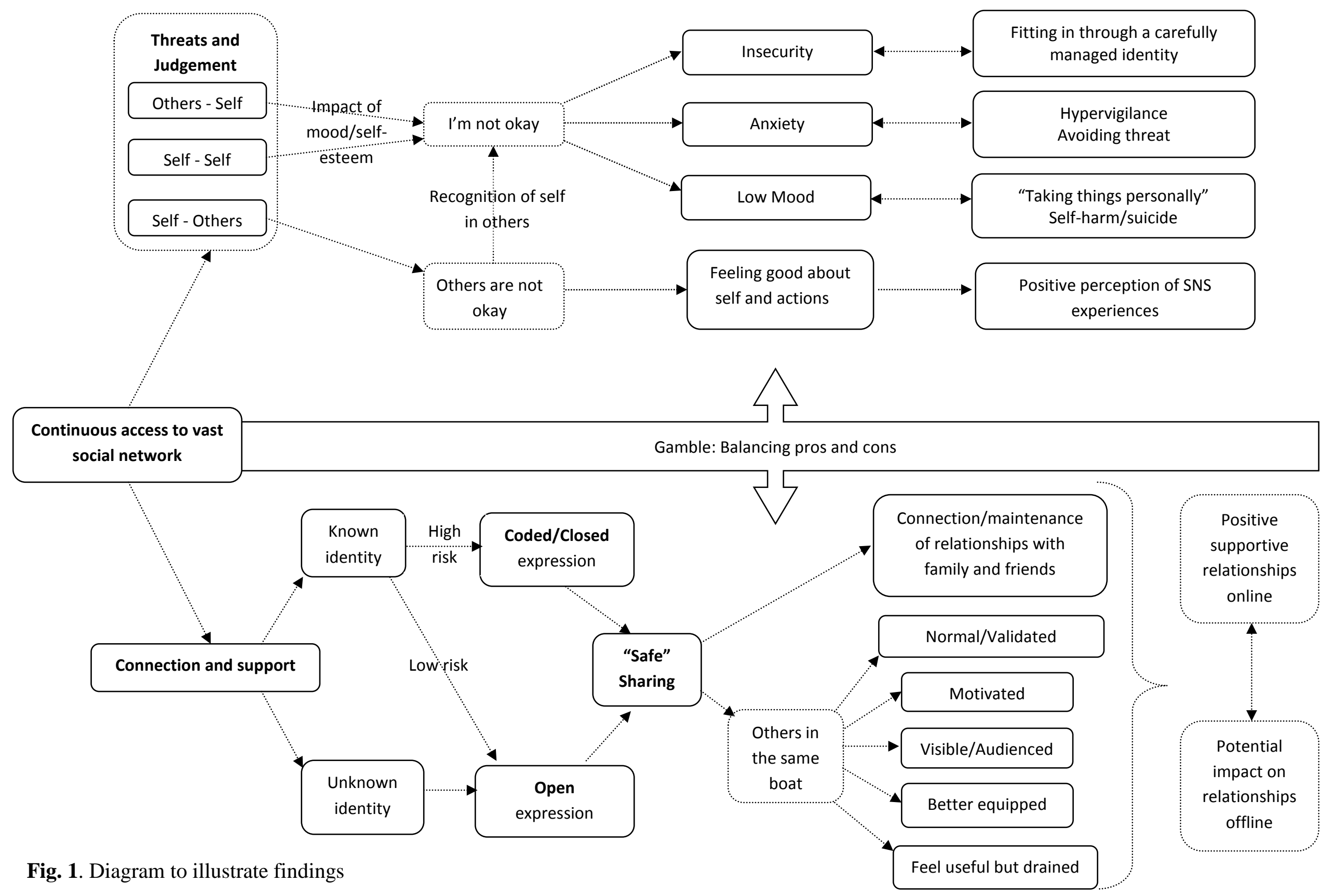




\subsection{Continuous access to a vast social network}

All of the young people described having constant access to the SNS that they used, many describing using the sites "continuously”. There was an inherent awareness of being part of a vast social network in which they were visible to friends, family and unknown others. This presented the challenge of being judged and receiving threats, as well as the opportunity for connection and support.

\subsection{Threats and judgement}

Underpinning many of the participants' experiences was judgement and the experience of SNS as a threatening place. Judgement was directed towards young people from others (others-self), from the young person towards themselves (self-self) and from the young people toward others (self-others). Across all experiences was the acknowledgement that existing mood, self-esteem and/or thinking styles influenced interpretation of threats on SNS, as Lizzie explained, "it can be really bad for people who have depression or mental illnesses because they can... just the way they work, makes them think differently from other people whereas someone might not bat an eyelid at something somebody’s said.”

\subsubsection{Threats and judgement: Others - self}

For most of the young people, judgement was received from those with whom they had offline contact, including peers at school. Nina (aged 13) described a graphic and upsetting threat from a peer who had become jealous after she expressed interest in another boy, "he said that he wants to like hang me and he wants to pour acid down my throat". Nina’s experience had led to frequent panic attacks, her reason for accessing CAMHS.

For some, judgement was specifically related to their mental health. Ben (aged 16) described, “I’ve had abuse, ‘you’re an Emo, you’re a freak, you're no good, you should just end your life instead of just saying that you want to’”. Similarly, Jane (aged 15) became 
fearful of others' response to her CAMHS attendance after witnessing a friend receiving judgement, "there was a boy in my school who posted 'I've got a CAMHS appointment tomorrow, I'm scared' and then people commented on his status saying, like, ‘you're a nutcase’”.

Judgement and threats were also received from unknown others, as Sarah described, “it’s like especially when you write your opinion on something like that, like everyone’s getting ready to criticise you and say you're wrong”. The young people were aware that interactions could be with strangers using false accounts, which too was interpreted as threatening, as Montague (aged 14) described, "I know friends who have had problems ...with a person who was stalking her... and it turned out to be one of these 40 year olds”.

Young people understood threats and judgement as a product of the features of communication on SNS. Receiving "likes" and "retweets", or the absence of these, was experienced as judgement about young people’s popularity, attractiveness or intelligence. Even when she received likes, Kim (aged 17) described how she felt she was, "still being judged, just 'cause it’s positive they're still thinking, and if they don't like your other picture, does that mean they didn't like you then?” The often intrusive nature of SNSs added to young people’s experience of threat. Ruby, a 17 year old girl described "never being safe”, as bullies from her school "followed her home” via her SNS. Additionally, young people recognised the power of anonymity on SNS, which meant that those who targeted the young people were separated from the social cues and responses that would be inherent in face-toface interactions, allowing them to become "keyboard warriors” (Sarah, aged 18).

\subsubsection{Threats and judgement: Self-self}

Young people viewed others’ pictures and personal information for prolonged periods of time. This encouraged an exaggerated version of offline social comparison processes; 
paying close attention to others' appearance and activities without breaking the usual face-toface social rules. Young people typically felt insecure when they noticed what they lacked in comparison to others, as Natalie (aged 15) described, “everyone’s gotta be better than everyone and if you're not better then you're nothing”. As an influential factor in most of their SNS interactions, young people identified that their mood influenced the nature of their comparison, as Ruby (aged 17) explained, “if you're feeling like particularly like selfconscious or something that day, you're more likely to pick up on other people looking better or skinnier or something which makes you feel worse about yourself”. Despite recognising the impact of such comparisons on their mood and wellbeing, young people found this process difficult to resist.

\subsubsection{Threats and judgement: Self-others}

Young people compared their own and others' use of SNS with considerable judgement. They identified people who expressed feeling low or suicidal, but who they felt were not genuine. Kim (aged 17) described her frustration towards such "attention seekers":

...it's like 'what are you doing?'...Like you're not going to do anything to yourself cause you're writing about it all over Twitter, you're wanting someone to stop you or you're wanting someone to be like 'oh don't babe', that's attention seeking, not being suicidal and self-harming, and that's what really frustrates me. I hate it.

Nevertheless, Kim went on to describe how when she feels low the number of tweets that she posts draws attention from friends at college, “[I’ll post] like 24 plus tweets and it’s like they're all like really depressing so then people read your thing and there's like, I've had people come up to me in college like 'was you having a bad day the other day?'”.

Young people described others’ posts as disgusting, unnecessary or deemed others as “pathetic” for needing reassurance through receiving "likes”, but equally valued likes 
themselves. Sally (aged 16) spoke of how others were dull and stupid for using SNS and that she was “not bothered” about others' lives, yet also spoke of her "compulsive” need to stay up to date with everyone’s statuses.

\subsection{I'm not okay}

Social comparison processes and direct judgement and threats from others typically led to young people’s belief they were "not okay", associated with insecurity, anxiety, and low mood. Young people implemented a range of strategies to cope with the emotional impact of this experience.

\subsubsection{Insecurity $\leftrightarrow$ Fitting in through a carefully managed identity}

Insecurity motivated young people to be wary and thoughtful about how they portrayed themselves online. They posted more frequently and openly when they felt happy or content, yet were more cryptic about their difficult experiences or distressing emotions.

Young people’s posting behaviour changed in response to validation or rejection from others. They analysed others' responses to their posts and considered how they might change to receive more "likes" in the future:

Say, for example, I upload two selfies, one gets 50 likes, the other one gets $5 . .$. what's wrong with me on that picture? Should I never have my hair like that? Should I never do my make up like that ever again? Am I chubbier in that picture? (Sarah, aged 18).

Such insecurity encouraged participants to present a "safe” and socially acceptable version of themselves, which in turn led to increased insecurity about how others might respond if they deviated from this careful presentation.

\subsubsection{Anxiety $\leftrightarrow$ Hypervigilance and avoiding threat}


Due to their experiences of being judged and receiving threats, young people felt anxious and vigilant to further threat and judgement. Sally described her "compulsive” need to ensure that there were no further threats to herself or others by checking her phone regularly throughout the interview:

If you've noticed I was holding my phone a lot cause it’s, it's like an addiction, I have to check them, so even if I just like read what they're saying there then that's fine, as long as I just see it to know what's going on, if it's something serious then I'll have to be like, 'oh I have to go or something'.

Participants frequently checked their newsfeed to ensure that they weren’t being spoken about despite the negative impact this could have on their mood. Many of the young people tried to delete or leave SNS altogether, but returned to satisfy their “curiosity”, as Sarah described, “someone will go ‘well why don’t you just block them’ but it’s not like that...it just takes over your brain, and you wanna know what they’re saying about ya” (Sarah). As leaving the sites was not a viable option for these young people, they often avoided further judgement by deleting posts or not posting at all, as Natalie described, "like, I wouldn’t wanna post no pictures, I wouldn’t wanna put a status just in case I draw attention to myself”. This strategy had a self-perpetuating effect, in that by attempting to avoid judgement altogether, participants became increasingly anxious about what would happen if they did then decide to post.

\subsubsection{Low mood $\leftrightarrow$ “Taking things personally” and self-harm}

Young people recognised how their experiences of being threatened or judged (by others or themselves) could lead to them feeling low in mood and "taking things personally":

if you look at something one day when you're feeling happy and it not have a negative impact on you at all, but if you look at it again the next day when you're 
feeling like quite low or something and...you'll sort of take things like a bit more sensitively... and you'll internalise it more than you would (Ruby)

For several young people, low mood was associated with self-harm as a means of coping. Seeing others' self-harm on SNS could be motivating, as Lizzie (aged 16) described, "it was sort of like it [self-harm] was an infectious disease because I knew they were doing it and...they felt better for it afterwards...it was like 'maybe I should do that'”. For Jane, witnessing others' self-harm similarly triggered her to harm, but in an attempt to validate her difficulties through matching the severity of others' self-harm.

\subsection{Others are not okay}

Through judging others, young people observed others' actions and interactions on SNS as inappropriate or unacceptable and felt better about themselves and their own actions.

\subsubsection{Feeling good about self and actions}

Judging others allowed young people to hold a more positive perception of their own SNS activities. For example, some young people perceived themselves as more able than others to navigate and cope with the challenges that SNS bring, "it doesn't hurt, doesn't hurt...you just ignore it but for some people whose confidence aren't as high as mine that's really damaging to them” (Ben).

Judging others also protected the young people from recognising in themselves the behaviours they disliked in others. For example, Nina recognised that her own actions could be considered bullying but explained how this was not reflective of her true character: 
I don’t know, I just felt a bit mean, I felt like a bully... and I don’t really like feeling like that, I always, if you ask any of my friends they'll tell ya 'she always apologises'...they’re like ‘you're too nice’. It’s not my fault.

Others, however, had begun to reflect upon the similarity between their own and others' actions, as Sarah described, "it has in the past moulded me into a narcissistic little bitch and I’ve suffered from that, I've been depressed ....As much as I don’t want to be shallow, like I'm going to get jealous”. By recognising this similarity, Sarah felt conflicted about her SNS interactions, contributing to her emotional experience of "I'm not okay".

\subsection{Connection and support}

All participants spoke of the benefits of SNS in connecting with others. The desire and, at times, duty to stay in touch with others was a powerful influence in young people's continued use of SNS and they expected that they would be excluded offline should they cease using the sites. All participants enjoyed meeting new people with whom they could talk and seek support from, providing they were able to achieve a level of anonymity that facilitated "safe sharing”.

\subsubsection{Unknown/known identity and safe sharing}

Young people valued having unknown others who they could contact or who would audience their posts, identifying Tumblr as a SNS on which they felt less identifiable and consequently more able to share due to the lack of personal details, perceived anonymity and the use of photographs and images as a means of communication. Ruby described how having an audience of strangers reduced her pressure to conform and relieved her anxiety about being excluded, “you're not as bothered about conforming cause you just think they don’t have an opinion on you anyway...they're not going to disown you or unfriend you”. 
Young people also considered strangers to be more "genuine” as Ben described, “your compliments off your friends they're saying that because they don't want to hurt your feelings, your family have got to be supportive”.

\subsubsection{Coded/open expression and safe sharing}

When young people's identity was known by others, they were careful about sharing information. Young people used a form of “code” when posting about their emotional experiences which increased their feeling of anonymity, often using song lyrics or photographs that portrayed their emotions, "maybe if you're feeling upset you could reblog loads of upset pictures, maybe if you're feeling dead happy you could reblog happy pictures” (Jules). Tumblr’s 'reblog' feature allowed young people to post others' pictures, perceived as safe as they did not share their own experience and thus reduced the possibility for judgement.

\subsection{Connection and maintenance of relationships with family and friends}

Connecting with family and close friends was largely described as a very positive experience for young people. Young people relied on particular friends who they knew would be able to have a positive influence on their mood, as Montague described: "if someone's taking the mick out of you on PlayStation for example, you can just go on Facebook, talk to a friend and y’know you're alright within seconds”.

Young people enjoyed arranging social activities over SNS, seeing photos of themselves with friends and staying connected with family who they rarely saw. Nevertheless, young people remained cautious about the offline repercussions of sharing openly, as Kim described, “four of my aunts are psychologists so anything I write they overanalyse it and then they phone my mum worrying”.

\subsection{Sharing with "others in the same boat"}


All of the adolescents in this study used SNS to seek contact and support from others who they perceived as similar to themselves, with a range of positive outcomes. For the majority, this was in relation to their emotional needs. The features of SNS facilitated this search, allowing young people to search using terms such as "sad”, finding support groups relating to specific difficulties, and looking up users who have created instructional videos about coping strategies.

\subsubsection{Feeling normal and validated}

Young people used SNS search features to find young people with similar difficulties who normalised and validated their emotional experiences. Ben described joining Facebook:

Yeah once I got Facebook this release it was overwhelming, cause when you're in your room on your own it's just you, that's all you see, everyone around you feels dead happy... when you go on Facebook you wake up, it’s like your eyes are opened because you notice that there's thousands all across the world in the same boat as you.

Features of SNS including likes and favourites, served to further increase young people’s feelings of validation and normalisation, as Sally described, “when you feel low you might put a song or some lyrics or something you can relate to, and there is that moral support part of it when people favourite you".

For some young people who struggled socially offline, SNS allowed an experience of having numerous friendships. Jane was able to have conversations that she would be unable to offline, which also helped her feel normal, “it’s good cause I don’t speak very much so it’s hard to find friends like in school and things but I can type and I don't have to open my mouth".

\subsubsection{Feeling motivated}


Contacting others in a similar situation also motivated young people to take positive action. Ruby sought support on SNS following a sexual assault. Although she struggled witnessing others' posts about such experiences, they enabled her to find the courage to go to the police. Natalie described feeling inspired by others, "these people have better lifestyles but it..I don’t like get jealous of them, they’re like, they inspire you [to] be happier”.

\subsubsection{Feeling visible/audienced}

Young people spoke of the benefits of simply being audienced about their personal struggles, independent of whether others would respond:

I have tried writing stuff down in a diary but I kind of get half way through and just rip it up and think 'no-one’s gonna ever read this so it doesn’t matter'...on Tumblr I know people are gonna see it (Jules).

They recognised how sharing feelings to unknown others was preferable as they would not take action in the same way as a parent or therapist might, as Kim described:

I didn't want to talk to [therapist] cause if it gets bad she has to tell my mum and stuff

like that, and my mum last time anything happens she locks like the knives away, locks all the doors and it's like 'wow, stop!'

\subsubsection{Feeling better equipped}

Connecting with people who had similar experiences also provided young people with coping strategies for managing their emotions. Jane described, "like I have a friend that if I'm triggered to hurt myself if I'm able to message her she helps distract me and talk me out of doing whatever I'm thinking of doing.”

Young people were aware of specific forms of support that they could access through features of SNS. Jules described a chat feature on Tumblr, "if you put in on the search bar 
'depressing pictures' then... it says 'if you are depressed or feeling suicidal, if you feel like you want to hurt yourself' and you can talk to... trained people”.

Several young people highlighted the benefits of educational videos that gave them information about anxiety management or alternatives to self-harm. Others had witnessed mental-health awareness promotions and coping strategies promoted online in relation to suicidal ideation.

\subsubsection{Feeling useful but drained}

In addition to receiving support, many of the young people used SNS to support others. Ben and Simon focused all their energies on offering others advice and felt a duty to do so. For Ben, seeing others who were experiencing difficulties made him feel "heartbroken”, “sad” and "guilty” for not helping and he felt a duty to help others. Others would “refer" young people to him who had posted images or videos of them self-harming and who they felt needed his support, increasing his duty to help.

Nevertheless, young people recognised that taking on others' difficulties might be a burden in addition to their own. Depending on their mood, others' negative experiences had the potential to make the helper feel worse, as Kim described, "instead of thinking 'well that's what they're going through, but I'm going through this right now, let's enjoy this moment' but you're not, you're always caught up in theirs”.

\subsection{Positive, supportive relationships online $\leftrightarrow$ Potential impact on relationships offline}

Young people reported their use of SNS facilitated a range of positive outcomes including feeling normal, validated, being better equipped to manage their psychological distress and ensuring that they maintained important relationships. For some who struggled socially offline, SNS connections provided them with community and belonging. Nevertheless, many of the young people expressed concern about how SNS affected their 
offline world. Primarily, young people recognised how their ability and confidence when interacting face to face was affected, "I know I can easily, I can have a conversation more easily with somebody if I'm typing it as opposed to verbally, as soon as that awkward silence sort of comes in it’s just like 'oooh I wanna leave now'” (Ruby). SNS use also influenced their daily social activities, "Cause a lot of people now are just not up for doing normal things like going out for a meal or something...” (Sally). All of the young people expressed concern about younger people, specifically siblings, who would be subject to similar threats as they grew up and who they recognised as struggling socially as a result of their SNS use.

Young people differentiated between offline and online forms of interaction, and used a variety of words to describe their offline interactions, such as "verbal”, "real life” or "face to face”. The eldest participant, Sarah, described her belief that a reliance on SNS relationships can lead to offline isolation, "obviously with the isolation comes mental illnesses as well, you know you don’t need to go out and socialise when you've got everyone at your fingertips, um...but actually, we are humans and we do need actual interaction”.

\subsection{Balancing pros and cons: The "gamble" of SNS use}

Young people's choice to remain on SNS highlighted its indispensable nature in their culture. They benefitted from their many positive experiences connecting with others and believed that reducing their SNS use would result in their exclusion from social circles offline. Furthermore, for many, their continued use was considered obligatory to enable their vigilance and preparation for future threats.

However, young people's constant uncertainty on SNS portrayed a sense of a “gamble”. Young people had developed strategies for minimising the risks of negative experiences yet still created posts with such and anxiety and uncertainty about what might be the response. Sarah experienced a high level of arousal in simply posting, "I suppose it’s 
quite daunting, can get your adrenalin going, can make you quite anxious”. When using SNS as a cure for boredom, young people clicked on things impulsively rather than making conscious decisions about how to use SNS, with each click representing a new gamble in the experience that might follow, "if you click on the wrong thing it can make you feel worse and worse and worse” (Ruby).

Nevertheless, despite this gamble-like uncertainty, Ben illustrated how, with enough caution, the young people could feel more in control of their SNS experiences:

Facebook isn’t bad, Facebook isn't good. It's a piece of software that's what people don't seem to realise, it's how you use that. If you mistreat that, it's not gonna be good, if you treat it with a bit of respect, use it respectfully, wisely, don't go mouthing your mouth off on it then you know you can meet some real decent people that you never could have met without it (Ben).

\section{Discussion}

For the young people in this study, SNS created a pressure to be constantly "switched on” to a vast social world where interactions with others were continuous. This world offered the potential for threats and judgement, with considerable negative impact on young people’s wellbeing. Influenced by their existing mood, self-esteem or cognitive processes, young people’s experience of threats and judgement on SNS increased feelings of anxiety, insecurity and low mood, which could be associated with coping behaviours such as selfharm. Nevertheless, SNS also provided positive reinforcing social experiences that validated and normalised emotional experiences and helped participants to feel included, worthwhile and better equipped to manage their distress. Young people’s positive experiences and avoidance of aversive experiences served to reinforce their use of SNS, which they described as "addictive”, or "compulsive”, at times affecting their offline activities and relationships. 
This conflict between the negative and positive aspects of SNS use echoes and augments some of the recent findings in recent quantitative research, such as that whilst SNS use increases “connectedness” it fails to reduce social isolation (Ahn \& Shin, 2013) and that active use of online support for psychological support helps reduce self-stigma whilst passive use of SNS prolongs it (Lawlor \& Kirakowski, 2014).

Young people recognised how anonymity allowed others to become "keyboard warriors": bullying or threatening them with directness and to an extreme not encountered offline, supporting other findings that anonymity corresponds with de-individuation and disinhibition in “cyberbullies” (Cassidy, Faucher, \& Jackson, 2013) and that young people are aware that others behave differently online (Ofcom, 2015). Cyberbullying has been found to have a particularly detrimental effect on adolescents with pre-existing emotional or psychological difficulties and can be associated with feelings of isolation, hopelessness and an increased likelihood of suicide attempts (Hinduja \& Patchin, 2010). The adolescents in this study highlighted how their negative experiences affected their mood, self-esteem, anxiety and their behaviours and relationships offline which in turn influenced their interpretations of such threats, highlighting the mutual relationship between their interpretation of threats and emotional wellbeing.

Social comparison processes are central to young people’s developing identities, enabling them to assimilate to social groups and fostering their understanding of themselves as being able to develop the skills to succeed in a way that is valued by society (Erikson, 1968). In this study, social comparison was perceived as an exaggerated version of offline comparisons as SNS allowed young people to look at others’ profiles for prolonged periods, without the social implications this would have offline. Adolescents predominantly made upward social comparisons which have been indicated to have a significant impact on selfesteem (Krayer, Ingledew, \& Iphofen, 2008), corroborated in this study in young people’s 
descriptions of themselves as "insecure”. Such findings may contribute to explaining the links found between SNS use and low self-esteem (e.g. Blachnio, Przepiorka, \& Pantic, 2016).

Adolescents in this study presented themselves carefully to "fit in" with those ideals they perceived as valued by their online social network. In accordance with theories of impression management, the aspects of self that received validation from others were maintained and incorporated into their online identities (Schlenker, 1986). Balick (2014) proposes that SNS identities are not "virtual" but representations of our multiple psyches responding to the imagined and real SNS audiences, allowing young people a forum for “identity-testing”. In this study, adolescents’ different SNS identities were carefully tried and tested with different audiences, and functioned to meet different needs. For example, they would seek emotional support through a more honest but anonymous version of themselves whilst maintain relationships with friends and family through a carefully portrayed public version of themselves. This anonymity seemed to enable the young people to side-step the difficulties identified in other research that has found adolescents’ online support-seeking to be diluted by an increased need for impression management (Oh \& LaRose, 2016).

Reflecting theories of cognitive dissonance, in which individuals strive for internal consistency and experience psychological distress when inconsistency (dissonance) occurs (Festinger, 1957), adolescents’ sought to reduce the dissonance between their beliefs and actions and achieve consonance by conceptualising their own support-seeking as genuine and much-needed, rather than "attention seeking”. Some young people were aware of this dissonance, however, and described feeling frustrated and conflicted about their actions.

\subsection{Limitations}


A limitation of this study is the small sample size. In classical applications of Grounded Theory, data collection would continue until "theoretical saturation”, described by Glaser (2001) as the “conceptualization of comparisons of these incidents which yield different properties of the pattern, until no new properties of the pattern emerge” (p. 191). In our case, whilst collecting additional data would no doubt have identified further insights, the fact that we were exploring subjective perceptions means that the categories that could be generated are potentially limitless. As O’Reilly \& Parker (2012) argue, in such situations achieving data saturation is unrealistic, but this in no way means that the findings are invalid. Rather we adopted Dey’s (1999) approach, which advocates for the collection of sufficient data to construct a theory that contains exemplar categories in key areas, allowing for an understanding of participants’ experiences. These can then be used to further develop and enhance theories for further testing and will provide useful materials for consideration by clinicians.

It is important to note that this study recruited predominantly female participants. The males who did participate shared differing experiences, notably a focus upon graphic sexual and self-injury images, a particular enjoyment in helping others, and relatively reduced social comparison processes compared with girls. As a result, the theoretical framework might not adequately address gender differences which have been recognised in the existing literature (Ferguson, 2013; Lin, Zhang, \& Li, 2016).

\subsection{Clinical implications}

Lewis et al. (2012) have developed specific guidelines and a sample interview schedule to enable clinicians to enquire about the impact of young people’s online experiences on self-injury behaviours specifically. A similar guideline relating to the impact of their SNS use more broadly might assist the consideration of such factors in assessment 
and formulation. This can then identify when interventions may be needed that can build upon young people's resilience to cope with their SNS experiences may be needed. Compassion-focused interventions (Gilbert, 2010) are one possibility in such circumstances, which might help young people foster a more compassionate position towards the self and others. Furthermore, young people’s behaviours of checking and regularly seeking reassurance by remaining hyper-vigilant to potential threat on SNS will be likely to maintain their anxiety about online experiences (Newman \& Borkovec, 1995). Clinicians might usefully work together with young people to understand how various strategies for coping with SNS positively or negatively influence their wellbeing or maintain distress.

For this group of young people, achieving a desired level of anonymity enabled them to seek support that they valued. Young people recognised that although mental health services offer support, there was a qualitatively different process occurring on SNS in which they felt able to connect with others with similar experiences who would not respond to their "risky" behaviours in a way that services or family might. This poses a challenge to many services in which a culture of "risk aversion" has developed to consider how such responses might serve to limit young people's ability to share their experiences and seek the support they need.

Whilst researchers suggest that many people prefer face-to-face support in relation to psychological difficulties, the vast majority will also access information and support online (Klein \& Cook, 2010). The reasons for this are multifaceted but include the option to remain anonymous and feeling less stigmatized (Johnsen, Rosenvinge, \& Gammon, 2002). SNS could be harnessed by services to provide evidence-based information or interventions whilst facilitating the anonymity and security that allows adolescents to be open about their experiences. 


\subsection{Future research}

As in previous research (Bryce \&Fraser, 2014), young people in our study undertook a conscious process of weighing up the potentially negative and positive outcomes of their SNS use. Their use of strategies allowed them to benefit from the positive aspects of SNS by navigating their negative experiences. These strategies differed in response to their mood and cognition, and dependent on the specific context. Future research might explore further the mechanisms by which young people select and employ various strategies and how these interact with psychological distress. Such research will also assist in explaining the links between distress, stigma, and social connection found in recent quantitative studies (e.g. Ahn \& Shin, 2013; Blachnio et al., 2016; Lawlor et al., 2014; Müller et al., 2016).

Although the young people in this study received positive support, many also described receiving direct abuse in relation to their psychological distress and some had witnessed the abuse of others who had publically admitted to accessing mental health services. Given the power of SNS in giving young people feedback about what is acceptable or not in their social world, research exploring the stigmatising messages that young people receive on SNS regarding their mental health might be helpful in understanding barriers to accessing support for young people.

Finally, authors often seek to highlight differences between online and offline relationships which might illustrate a societal privilege that we assign to "real world" connections over friendships online. However, the value and importance of the adolescents' online relationships in this study was striking, with many perceiving their online relationships as equally "real” to those offline. Furthermore, many relationships identified by participants had both an "online" and "offline” component, blurring such distinctions. Further research 
could explore adolescents’ perspectives about the value of online and offline relationships, and whether a clear separation of the two exists for them. 


\section{References}

Ahn, D., Shin, D-H. (2013). Is the social use of media for seeking connectedness or for avoiding social isolation? Mechanisms underlying media use and subjective well-being. Computers in Human Behavior 29(6), 2453-2462.

Anderson, B., Fagan, P., Woodnutt, T., \& Chamorro-Premuzic, T. (2012). Facebook psychology: Popular questions answered by research. Psychology of Popular Media Culture, 1(1), 23-37.

Balick, A. (2014). The psychodynamics of social networking: Connected-up instantaneous culture and the self. London: Karnac Books Ltd.

Błachnio, A., Przepiorka, A., \& Pantic, I. (2016). Association between Facebook addiction, self-esteem and life satisfaction: A cross-sectional study. Computers in Human Behavior, 55, 701-705.

Bretherton, I., Munholland, K., Cassidy, J., \& Shaver, P. (1999). Internal working models in attachment relationships: A construct revisited. In J. Cassidy \& P. Shaver (Eds.), Handbook of attachment: Theory, research, and clinical applications (pp. 89-111). London: The Guilford Press.

Bryce, J., \& Fraser, J. (2014). The role of disclosure of personal information in the evaluation of risk and trust in young peoples’ online interactions. Computers in Human Behavior, 30, 299-306.

Cash, S. J., Thelwall, M., Peck, S. N., Ferrell, J. Z., \& Bridge, J. A. (2013). Adolescent suicide statements on MySpace. Cyberpsychology, Behavior and Social Networking, 16(3), 166-174. 
Cassidy, W., Faucher, C., \& Jackson, M. (2013). Cyberbullying among youth: A comprehensive review of current international research and its implications and application to policy and practice. School Psychology International, 34(6), 575-612.

Charmaz, K. (2006). Constructing grounded theory (1st Edn.). London: Sage Publications Ltd.

Chou, H., \& Edge, N. (2012). “They are happier and having better lives than I am”: The impact of using Facebook on perceptions of others’ lives. Cyberpsychology, Behavior, and Social Networking, 15(2), 117-120.

Chu, P. Sen, Saucier, D. A., \& Hafner, E. (2010). Meta-analysis of the relationships between social support and well-being in children and adolescents. Journal of Social and Clinical Psychology, 29(6), 624-645.

Collin, P., Rahilly, K., Richardson, I., \& Third, A. (2011). The benefits of social networking services. Retrieved from http://researchrepository.murdoch.edu.au/11804/

Dey, I. (1999). Grounding grounded theory. San Diego: Academic Press.

Erikson, E. H. (1968). Identity: Youth and crisis. New York: Norton.

Feinstein, B. A., Hershenberg, R., Bhatia, V., Latack, J. A., Meuwly, N., \& Davila, J. (2013). Negative social comparison on Facebook and depressive symptoms: Rumination as a mechanism. Psychology of Popular Media Culture, 2(3), 161-170.

Ferguson, C. J. (2013). In the eye of the beholder: Thin-ideal media affects some, but not most, viewers in a meta-analytic review of body dissatisfaction in women and men. Psychology of Popular Media Culture, 2(1), 20-37. 
Festinger, L. (1957). A theory of cognitive dissonance. Stanford, CA: Stanford University Press.

Gilbert, P. (2010). The compassionate mind (1st ed.). London: Constable and Robinson Ltd.

Glaser, B. (2001). The grounded theory perspective: Conceptualization contrasted with description. Mill Valley, CA: Sociology Press.

Gosling, S. D., Augustine, A., Vazire, S., Holtzman, N., \& Gaddis, S. (2011). Manifestations of personality in Online Social Networks: Self-reported Facebook-related behaviors and observable profile information. Cyberpsychology, Behavior and Social Networking, 14(9), 483-8.

Hamm, M. P., Newton, A. S., Chisholm, A., Shulhan, J., Milne, A., Sundar, P.,...Hartling., L., (2015). Prevalence and effect of cyberbullying on children and young people: A scoping review of social media studies. JAMA Pediatrics. 169(8):770-777.

Hinduja, S., \& Patchin, J. W. (2010). Bullying, cyberbullying, and suicide. Archives of Suicide Research, 14(3), 206-21.

Johnsen, J.A. K., Rosenvinge, J. H., \& Gammon, D. (2002). Online group interaction and mental health: An analysis of three online discussion forums. Scandinavian Journal of Psychology, 43(5), 445-449.

Kim, J., LaRose, R., \& Peng, W. (2009). Loneliness as the cause and the effect of problematic Internet use: The relationship between Internet use and psychological wellbeing. Cyberpsychology \& Behavior, 12(4), 451-455.

Klein, B., \& Cook, S. (2010). Preferences for e-mental health services amongst an online Australian sample. E-Journal of Applied Psychology, 6(1), 27-38. 
Krayer, A., Ingledew, D. K., \& Iphofen, R. (2008). Social comparison and body image in adolescence: A grounded theory approach. Health Education Research, 23(5), 892-903.

Lawlor, A., \& Kirakowski, J. (2014) Online support groups for mental health: A space for challenging self-stigma or a means of social avoidance? Computers in Human Behavior 32, $152-161$.

Lewis, S. P., Heath, N. L., Michal, N. J., \& Duggan, J. M. (2012). Non-suicidal self-injury, youth, and the Internet: What mental health professionals need to know. Child and Adolescent Psychiatry and Mental Health, 6(1), 13-22.

Lin, X., Zhang, D., \& Li, Y. (2016). Delineating the dimensions of social support on social networking sites and their effects: A comparative model. Computers in Human Behavior $58,421-430$.

Merikangas, K. R., He, J., Burstein, M., Swanson, S. A., Avenevoli, S., Cui, L., Benjet, C., Georgiades, K., Swendsen, J. (2010). Lifetime Prevalence of Mental Disorders in US Adolescents: Results from the National Comorbidity Study-Adolescent Supplement (NCS-A). Journal of the American Academy of Child and Adolescent Psychiatry, 49(10), 980-989.

Muise, A., Christofides, E., \& Desmarais, S. (2009). More information than you ever wanted: Does Facebook bring out the green-eyed monster of jealousy? Cyberpsychology \& Behavior, 12(4), 441-444.

Müller, K. W., Dreier, M., Beutel, M. E., Duven, E., Giralt, S., \& Wölfling, K. (2016). A hidden type of internet addiction? Intense and addictive use of social networking sites in adolescents. Computers in Human Behavior 55 Part A, 172-177. 
Newman, M., \& Borkovec, T. (1995). Cognitive-behavioral treatment of generalized anxiety disorder. The Clinical Psychologist, 48(4), 5-7.

O’Keeffe, G. S., \& Clarke-Pearson, K. (2011). The impact of social media on children, adolescents, and families. Pediatrics, 127(4), 800-804.

O'Reilly, M., \& Parker, N. (2012). ‘Unsatisfactory Saturation’: a critical exploration of the notion of saturated sample sizes in qualitative research. Qualitative Research 13(2), 190197.

Ofcom. (2013). Children and Parents: Media Use and Attitudes Report. Retrieved from http://stakeholders.ofcom.org.uk/binaries/research/media-literacy/october2013/research07Oct2013.pdf

Ofcom. (2015). Children and Parents: Media Use and Attitudes Report. Retrieved from http://stakeholders.ofcom.org.uk/market-data-research/other/researchpublications/childrens/children-parents-nov-15/

Oh, H. J., \& LaRose, R. (2016). Impression management concerns and support-seeking behavior on social network sites. Computers in Human Behavior 57, 38-47.

Parker, P. D., Ciarrochi, J., Heaven, P., Marshall, S., Sahdra, B., \& Kiuru, N. (2015). Hope, Friends, and Subjective Well-Being: A Social Network Approach to Peer Group Contextual Effects. Child Development, 86(2), 642-650.

Quinn, S., \& Oldmeadow, J. a. (2013). Is the igeneration a “we” generation? Social networking use among 9- to 13-year-olds and belonging. The British Journal of Developmental Psychology, 31(1), 136-142. 
Rafnsson, S., Shankar, A., Steptoe, A. (2015). Longitudinal Influences of Social Network Characteristics on Subjective Well-Being of Older Adults: Findings From the ELSA Study. Journal of Aging Health, 27(5), 919-934

Schlenker B.(1986). Self-identification: Toward the integration of the private and public self. In Baumeister R.F., (Ed), Public self and private self (p. 21-62).. NewYork, NY: Springer-Verlag.

Spies Shapiro, L. A., \& Margolin, G. (2013). Growing up wired: Social networking sites and adolescent psychosocial development. Clinical Child and Family Psychology Review, 17(1), 1-18.

Staksrud, E., Ólafsson, K., \& Livingstone, S. (2013). Does the use of social networking sites increase children's risk of harm? Computers in Human Behavior, 29(1), 40-50.

Steinberg, L. (2010). Adolescence (9th Edn.). Boston, MA: McGraw Hill.

Subrahmanyam, K., \& Greenfield, P. (2008). Online communication and adolescent relationships. The Future of Children, 18(1), 119-146.

Suler, J. (2004). The online disinhibition effect. CyberPsychology and Behavior, 7 (3), 321326.

Valkenburg, P. M., \& Peter, J. (2011). Online communication among adolescents: An integrated model of its attraction, opportunities, and risks. Journal of Adolescent Health, 48(2), 121-127. 